Servicio de Tiviclogía

"Hospits1 Alrrioràn".

Sanatorio "Los Guindos"

del Consejo de Defensa del Niño.

\title{
LA ATELECTASIA PULMONAR EN EL NIÑO Y SUS RELACIONES CON LA TUBERCULOSIS PRIMARIA
}

(Continaación)

\section{Por los Dres. JORGE PEÑA CERECEDA, ERNESTINA PEÑA D. Y LUCILA CAPDEVILLE.}

\begin{abstract}
OBSERVACION CLINICA N. 2.-Servicio de Tubereulosis 5009.-4. Artỉián.

I. S.-.-1 año 9 meses, Ingreso: $5-X-42$.

ANTECEDENTES.—Madre tubercuiosa. No acusa antecedentes persinaLes,
\end{abstract}

ENFERMEDAD ACTUAL.-Desds hace dos meses, proenta tos. espriración quejumbrosa y decaimiento en forma intermitente.

Al examen genzal se encuentra pálida, decaida. Afabril.

Peso: $6.700 \mathrm{~kg}$. Sedimintación. $22.39 \mathrm{~mm} / \mathrm{h}$. Pitquet: Positivo.

Al examen palmooat. encontramos macidez $y$ respiración soplante en il campo izquierdo. Estertores gruesos y medianos en el tercio inferior derecho. La radiografia 36778, del 6-X-42 (figura 3-1), muestra una sombra homogéna, densa, que ocupa sasi todo el hemitórax izquiecdo, dejando libre un prequeñon espacio en el vértice. Ligera desviación dal mediastiro a la izquierda.

Continúa con cos. Ligeras febriculas. Rinofaringitis lecies con frecuen is. El examen palmonar no presenta variaciones.

BRONCOSCOPIA.-3-XI-42. Tráquea y bronquios con secreción muit; purulenta abundance. A nivel de la bifurcación se ve la mucosa roja y al panecer ligeramente tumefacta, sobre rodo a nivel de la embocadura del bronouic izquierdo. No es purden dar más detalles oor intranquilictad de la niña. (Dr. Parada).

Radiografia 37442 del $16-X I-42$ (figura $3-2$ ). Se observa ton aumento de la sombra descrita, tanto en intensidad como en extansión. que ahora rupa la totalidad del hemitórax. 
Contrae una coquelucbe en el Servicio, que agrava el cuadro con la aparición de fiebre alta, tos y edema de da cara. El examen clínico puimonar da sibilancias en ambos campos. No se logra apteciat otros signos. Después de 16 días de diagnosticada Ia coqueluche, fallece.

Se envía a la autopsia con el diagnóstico de Coqueluche, Atelectasia. Narmoria caseosa?.

AUTOPSIA 596 (Dra, E. Prña).

Examen macroscópico.-Deformación coxáxica conistente en solevantamirnto $y$ abcmbamiento del hemitórax izquizrdo, especialmente en la región aytzroJatexal inferior. Plentsia putulenta enquistada izquierda, con piduipleuritis fibrosa adhesiva de toda la sara externa (200 cc.) con pus algo espeso, amarillo verdoso. (Fig. 3-3).

Bronquitis aguda mucopurulenta, Atelectasia crónica. Nepmonia eróvica de la totalidad del fulmón izquizzdo. Enfisema casi total, y zonitas de atelectasia por obstración brorquial del borde anteroinfetior del lobulo medio $y$ de la base del puimón derecho. (Pulmón de aspecto coquatuchoso).

Hiperplasia discreta y tuberculosis cascosa parcial de un ganglio intertráquecbrónquito izquierdo, situado entre las primeras ramificaciones de los bronquios mayores.

En la autopsia se compruaba que no bay obstrucciön, retracción, ni coms presión bronquial por el ganglio antes descrito, sino que la compresión se ba cjercido probablemente por ganglios paratraquezles izquierdos, de! volumen de un frejol, hiperémicos y por el derrame pleural ga menciopado.

Nota.-En este cadaver no se ha encontrado macroscópicamente lesiones de tuberculesis n las pulmonss, ni tampoco generalizacicnes tuberculosas miliare:

Liquido plabal.-investigación de neumozoco negativa. Estaba invadido por los Proteus $y$ demís gérmenzs ádavéricos.

Examen microscópico.-Pleura parietal izquictuda. Hay un engrosamiento fibroso de la plenra que alcanza casi medio centímetro de espesor, formado por tcjido conjuntivo fibroso adulto, de gruesas fibras entrecruzadas \& infiltradas de linfecitos en forma difusa. siendo más infensa esta infiltración a mivel de Jos vasos.

Hay zonas numerosas con vasos de neoformación llenos de sangte $y$ en $\mathbf{1}$ superficic de th pleura hay, en generat, na necrozis del tejido, en la que perd sisten aigunos vascs de neoformación llenos de angre e infiltración linfocicaris intensa de algunas zonas y ent muchas otzas presencia de polinacleares degerivados.

Resumiendo.--En esta pleura hay lesiones crónicas de pąuipleturitis $\nabla$ saperficialmente una pleuricis aguda puralenta. En el carî examinado no cncontramos lesicnes tuberculosas.

Pajmón izquierdo--Corte de la cars lateral y superior vecina al vértice. - Tin escas zonas encontramos también lesiones de paquipletutitis que en uno de us bordis etetí en franca organización $y$ en es otre. las trsiones sor idénticas 


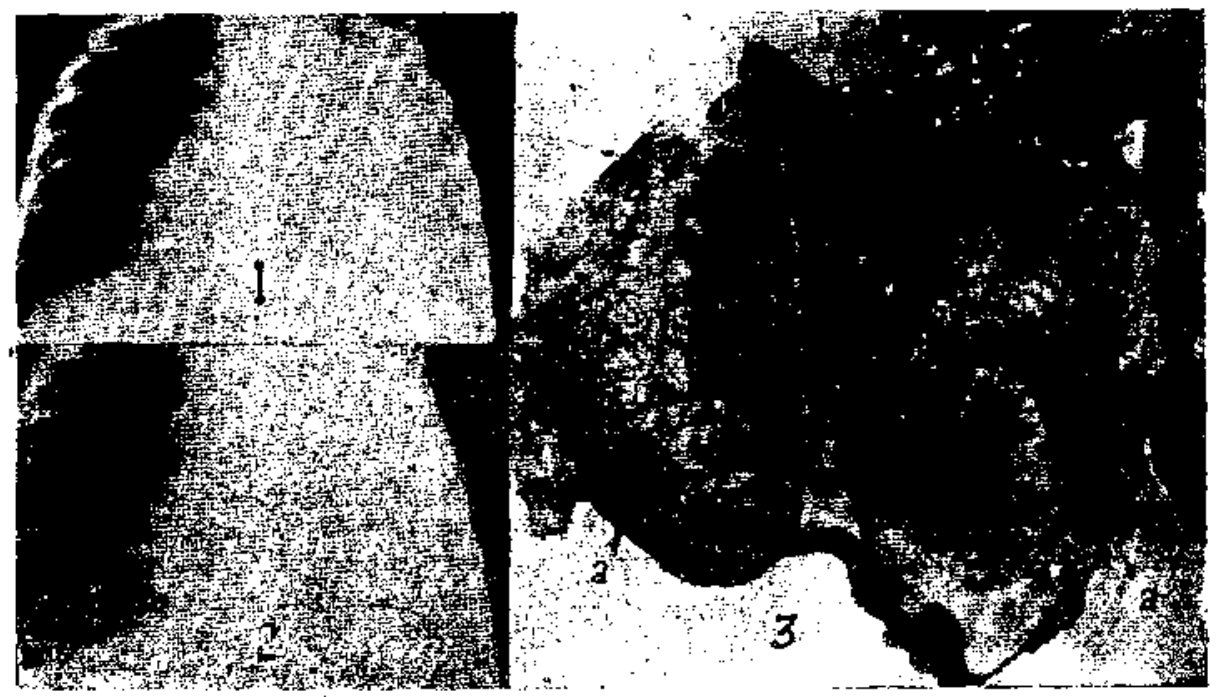

FIGURA N. 3. - ORSERVACION CLINTCA N: 2

1) Atelectasia de casi todo el bamitórax izquierdo. Queda ibre una pequeña zona er el vérice. Mediastino desviado a la izquierda. 2) La sombra be ha extendido a todo el bemitórax y se ha hecho más densa. 3) a) Se aprecia la paquipleuritis $y$ la cavidad del empiema; b) Al corte puede verse la atelectasia de un color mas oscuro.
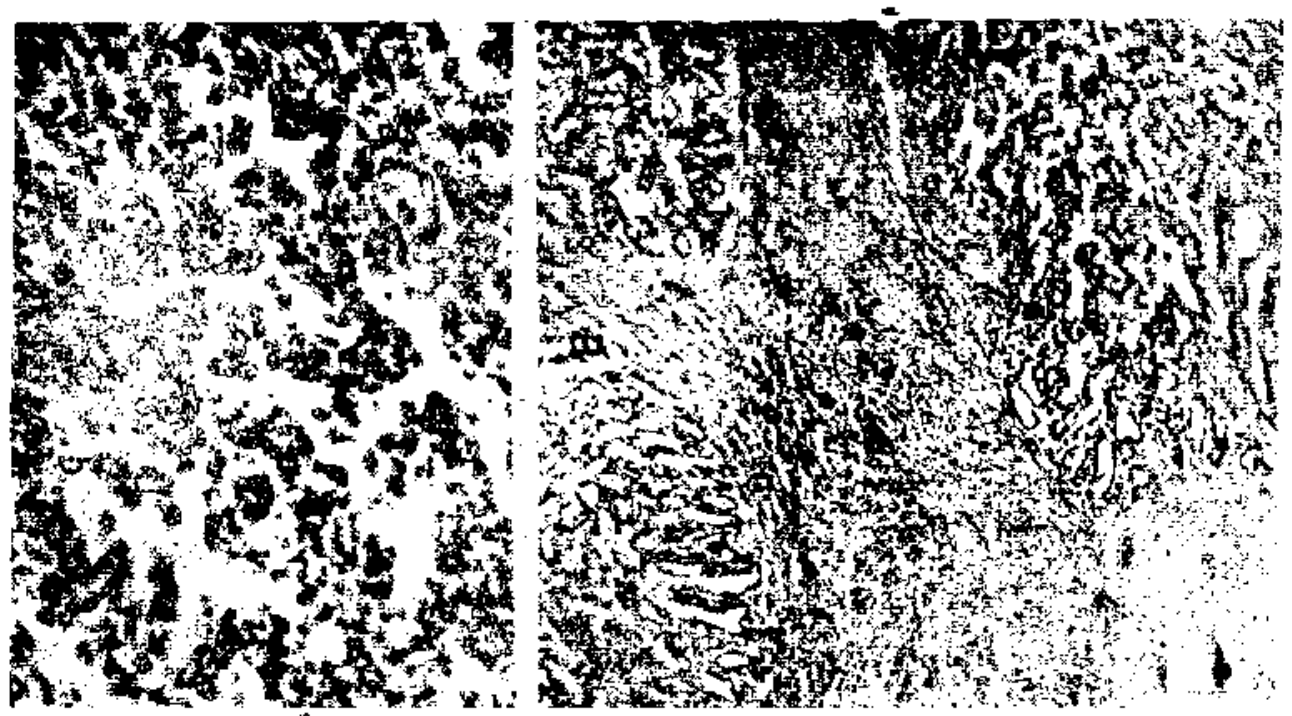

FIGLRA N." 4. - OBSERVACION N:? 2

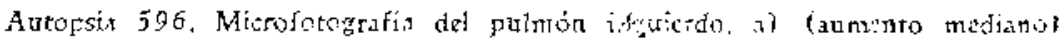

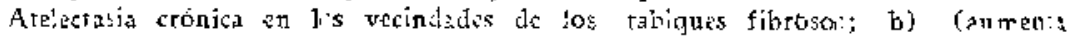
mavat). Se aprecia el epicelio cubia do los alvéolos. 
a las descritas en la pleuca parietal. Tampoco bemos encontrado en la pieara lesiones de tuberculosis. Esta preparación, observada con aumento menor, tien? una disposición lobulillar constituída por verdaderos tabiques fibrosos que parten de la pleura, dirigiéndose bacia el interior. Hay bandas fibrozas formatas por tejido conjuntive adulto $y$, además, bay fibrillas g pasos numerosos de jaytejes delgadas, repletos de sangre.

Estudiando estos lobulillos, constituídos propiamenta por alvéolos, encontramos ias leviones siguientes: los aivéolos escán siluados inmadiatamerte por debajo de la pleura $y$ muchos también en el interior del cette; pero. Jos que eatán en relación con los ábiquis fibrosos ya descritos, aparecen plegatus y semiplegados, Se epitalio es francamente eúbico y todos ellos se observan $v a$ cios (Figz, 4 a y b).

Hay oitos alvéolos pleg'ados o semiplegados. de epitelio plano. que tambiér están vacics. En orras zonas bay de gofferencia alvéolos que contienzr. atagte, linforitos, células indotelides descamadas escasas y líquido de edema. Los alvéolos ion edema puro son escasos. Por hiltimc hay alvéolos que contic nen únicamence célalas endotelizles descamadas de la paxed alveolar, algunas de las cualıs están hiprerplasiadas, pero en general con sus núlecis biar co'oreados. En muchas paredes alviolares hay capilares dilatados, repiptos de sangre. En este corte, no cucontramos lesiones de enfisema.

Bronquios,--En el corte hay pequeños y madianos; unos y otros presintan lesiones de bronquitis aguda puruienta y lesicnes de peribronquitis.

Resumiendo.-Aparte de las lesiones de la pleuta ya mizcionadas, encontramos en el parénguima pulmonar, lesione; francas de atelectasia. que pod: mos catalcgar de crónicas y lesiones polimorfas de alycolitis descanativa. hiporémica, edematosa e inflamaroria $y$ además lesiones agutas de bronquités $y$ peribrongutitis como se observa en la coqueluche.

En genetal, las paredes alveolates prosentan un aumento de tejido cin. juntivo fibroso, que es más denso en algunas zonas, esperialmente en las w. cinas a los tabiques fibrojos antas mencionados $y$ a los bronquios.

CONCLUSION-Pleuresía purulenta enquistada izquierds. Paquiplexristis fibrosa adhesiva izquierda, Atejectasia crónica.

Comenitario.-En esta niña debemos considerar dos aspectos fundamentales pata precisar la patcgenia de la atsletasia. Por una parte el empiema del lado izquierdo y por otca. el exudado mucopurulento del árbol bronquial. No creemess que los ganglíos descritos en el protocolo de autopsia hayan desemperiado un papel de importancia en la producción de ia atelectasia, porque su tamaño era relativamente pequeño y $\leqslant 11$ situación en las ramificaciones de los bronquios mayores no explica la producción de una atelectasia masiva como la observada en este caso. La secreción mucopurulenta y las lesiones de bronquitis y peribronquitis aguda corresponden seguramente a la coqueluche. 
Queda por considerar el papel que ha desenipeñado en ia patogenía de la atelectasia el empiema pleural. Dada su ex. tensión y su localización, creemos que el empiena ha determinado' una atelectasia por compresión, que podriamos íncluir en el grupo de las 1lamadas amyziras por Woatenhoefer (18) y sobre las que ya nos hemos treferido más arriba. 